\title{
Anisotropy of the Space Orientation of Radio Sources. I: The Catalog
}

\author{
V. R. Amirkhanyan ${ }^{1}$ \\ ${ }^{1}$ Sternberg Astronomical Institute, Universitetskii pr. 13, Moscow, 119992 Russia
}

(Received January 21, 2009; Revised May 20, 2009)

\begin{abstract}
A catalog of extended extragalactic radio sources consisting of 10461 objects is compiled based on the list of radio sources of the FIRST survey. A total of 1801 objects are identified with galaxies and quasars of the SDSS survey and the Veron-Veron catalog. The distribution of position angles of the axes of radio sources from the catalog is determined, and the probability that this distribution is equiprobable is shown to be less than $10^{-7}$. This result implies that at $\mathrm{Z}$ equal to or smaller than 0.5 , the spatial orientation of the axes of radio sources is anisotropic at a statistically significant level.
\end{abstract}

1. INTRODUCTION

The uniform morphology of galaxies make them excellent test bodies for the investigations of the structure of space. The British astronomer Brown was the first to study the orientation of galaxies in 1930s [1]. Brown [2], Nilson [3], Lauberts [4], and Karachentsev et al. [5] compiled extensive catalogs providing the galaxy parameters that are of most importance to such studies: position angles and axial ratios. Reinhardt [6], Nilson [7], Lauberts [4], Mandzhos [8], and Parnovsky et al. [9] analyzed these catalogs and showed convincingly that the spatial orientation of galaxies is anisotropic on the scale lengths of at least $200 \mathrm{Mpc}$. Extended extragalactic radio sources can be seen to exhibit equally uniform and anisotropic structure. Radio sources are very appealing objects for such studies, because they should eventually allow to either prove or refute the results based on an independent sample of galaxies and increase substantially the volume of the space domain studied. Amirkhanyan [10] tried to use such radio sources as indicators of anisotropy, but failed to reach a conclusive result because of a rather small size of the available homogeneous sample, which consisted of 298 radio sources of the MG catalog. It is evident that to obtain a statistically significant result, a catalog of extended radio sources is required, comparable in size with galaxy catalogs. However, no such catalogs are available to date. Therefore, we decided, with the ultimate aim of analyzing the spatial orientation of radio sources, to compile such a catalog based on the list of objects of the FIRST survey [11]. This catalog cannot be used directly since it provides no information about the multiplicity of radio sources. The catalog gives the parameters of individual components without indicating eventual associations between them.

\section{THE CATALOG}

\subsection{Rules of Selection}

For the task to succeed, minimizing the number of false objects is more important than finding all the true radio sources. Hence, the selection rules embedded into the first01 program that produces the catalog, focused on the former rather than the latter goal. The rules are as follows:

(1) each radio source must consist of at least three components;

(2) the integrated flux from each component must be greater than or equal to $2.5 \mathrm{mJy}$;

(3) the root mean square distance of the components from the axis of the radio source drawn optimally across the coordinates of the components must not exceed $0.12 \Theta$, where $\Theta$ is the separation between the most distant components.

We use the cluster analysis methods to generate the list of components that make up the radio source. Under these conditions, if we assume that all the 440046 objects of the FIRST survey with integrated fluxes equal to or greater than $2.5 \mathrm{mJy}$ are mutually unassociated, we should find no more than three false radio sources with three components. The probability of finding a false object with four or more components is negligible. Another possible source of false objects are very extended radio sources, where the separation between the groups of components exceeds the clusterization radius of $60^{\prime \prime}$. In this case, the program may mistake a group of components, if it meets the above rules of selection, for an independent radio source. Such objects are rare and most of them do not satisfy the rules of selection. The program yielded a catalog consisting of 10461 radio sources. Figure 1 shows examples of objects combined and their radio images in the FIRST survey. We performed such visual control for several tens of radio sources and it revealed no errors whatsoever. The program simultaneously identified the radio sources combined with objects of the SDSS survey [12] and the Veron-Veron Catalog [13]. If the separation between the average coordinates of the 
(a)
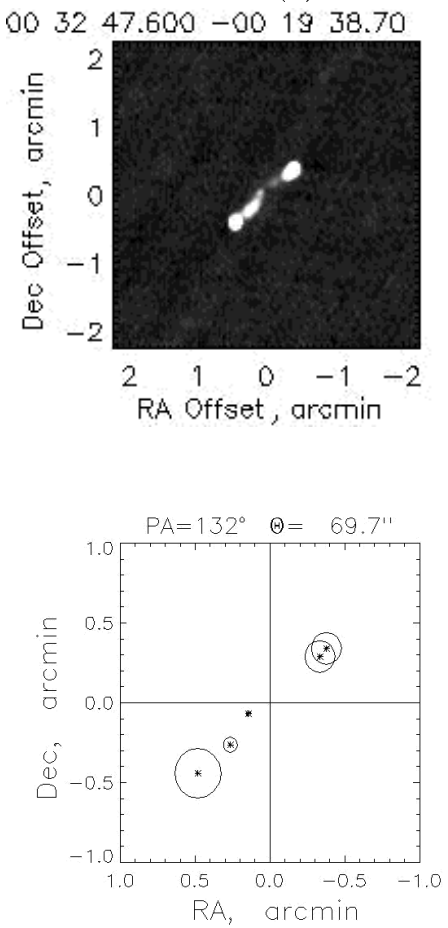

(b)
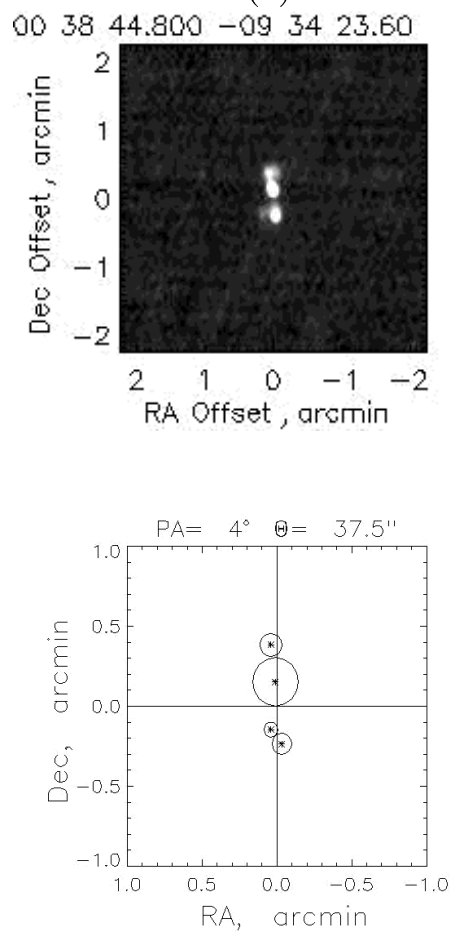

(c)
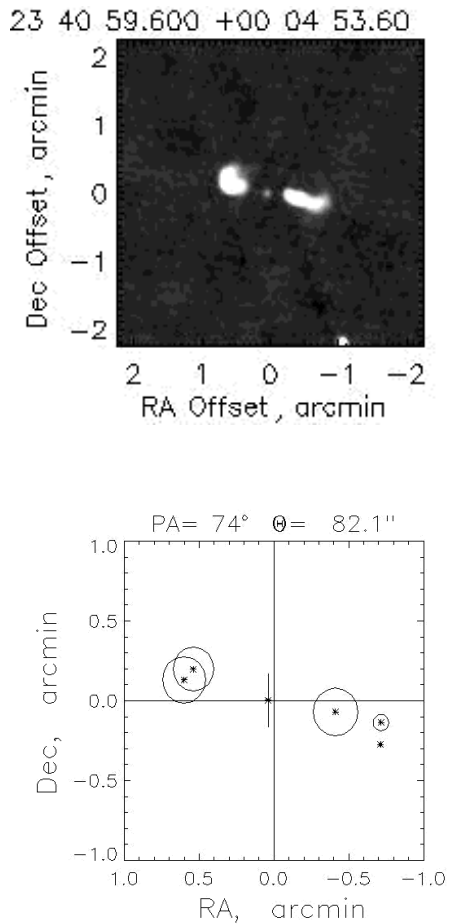

Figure 1. Images of radio sources in the FIRST survey (the upper line) and in this catalog (the lower line). The intersection of the $\alpha$ and $\delta$ axes shown on the maps of the lower line coincides with the average coordinates of the combined radio source. The asterisks indicate the positions of the components, and the diameters of the rings surrounding the asterisks are proportional to the integrated fluxes of the components. The cross indicates the location of the optical component (the figure on the right).

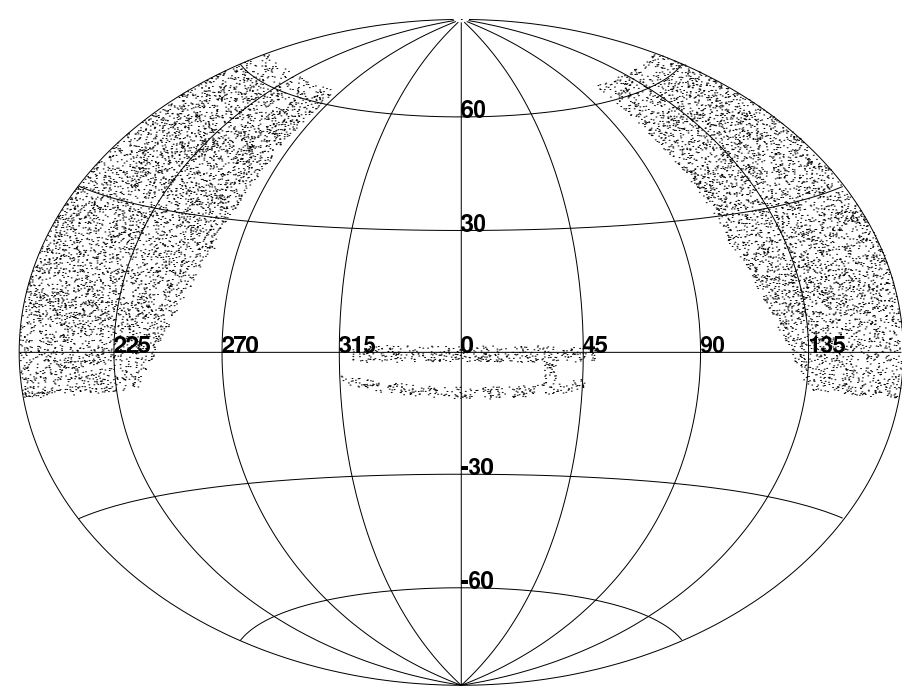

Figure 2. Sky distribution of the radio sources of the catalog.

radio source and optical object was less than $30^{\prime \prime}$ and less than half the angular size of radio source, the object was included into the catalog as a possible optical component. We then determine the component of the radio source that is nearest to the optical object, and compute the separation between them. At the same, we compute the distance from the optical object to the axis of the radio source. In subsequent studies we considered the radio source to have an optical identification if at least one of these separations was less than $3^{\prime \prime}$. A total of 1801 sources meet this condition. 


\subsection{Simulation of the Catalog}

Visual control is limited in scope and subjective in nature. Therefore, to test the quality of the search program, we generated several simulations of the FIRST survey. The sim_first01 code generated the preset number of extended radio sources in accordance with the given sky distribution. The number of components in radio sources was set in a random way from three to ten. To make the model as realistic as possible, we chose the distributions of fluxes, angular sizes, and scatter of components about the axis of the object to be close to the corresponding distributions in the real catalog. Position angle is the most important parameter of radio sources for our task, and therefore the simulation code allowed the form of the given distribution of position angles to be changed so as to compare it with the position angles of simulated objects. We number this list as 1. After completing the simulation, the program generated the combined list of the components of all simulated radio sources and wrote their coordinates and fluxes into a file in the format of the FIRST catalog (list No. 2). This file was then processed by the first01 program, which composed list No. 3 consisting of simulated objects. A comparison of lists Nos. 1 and 3 for several simulations showed that about $8.5 \%$ of the objects of list No. 1 fail to make it into list No. 3, since the scatter of their components about the axis exceeds the preset threshold (selection rule No. 3). Furthermore, about $4 \%$ of all objects are discarded as the separation between the groups of their components exceeds the clusterization radius. No false objects lacking in list No. 1 have been found in list No. 3. Reduction of simulated catalogs revealed a serious problem to arise when one has to compute the position angle of the axis of a radio source in case where this angle is close to 0 or 90 degrees. This problem is easy to explain.
Let $x_{i}$ and $y_{i}$ be the coordinates of $N$ components with respect to the center of mass of the radio source. The denominator or numerator of the formula for the slope of the straight line drawn through the set of points using the least squares method always contains $\sum_{i=1}^{N} x_{i}^{2}$ or $\sum_{i=1}^{N} y_{i}^{2}$. The coordinates of the components always include measurement errors, and therefore the above sums obey the $\chi^{2}$ distribution, and the probability that they should reduce to zero is negligible. Hence the probability to correctly estimate the position angle decreases as the real position angle approaches to 0 or 90 degrees (depending on the method used to draw the line) and, consequently, the number of objects of the histogram at these positions should also steadily decrease. As a result, we obtain a distorted distribution of position angles. To overcome these limitations, the program determines each position angle via a two-stage process. First, it uses standard formulas to find the straight line with the lowest sum of squared distances from the components, and computes its position angle $\phi$. The program then refines the position angle by varying the slope of the line within $\phi \pm 10^{\circ}$, and seeks the real minimum of squared distances. Numerical simulations showed that this computation method eliminates the problem of finding the position angle: the errors between the position angles of simulated (list No. 1) and combined (list No. 3) objects do not depend on the angle value, and the forms of the preset and measured position angle distributions of the objects from the simulated catalog coincide.

\subsection{Format of the Catalog}

The final catalog of extended radio sources has the layout as described in the Table.

Table. Extended radio sources

\begin{tabular}{c|c|c|c|c|c}
\hline$\alpha_{2000}$ & $\delta_{2000}$ & \multicolumn{4}{|c}{ Characteristics of the source } \\
\hline$(1)$ & $(2)$ & $(3)$ & $(4)$ & $(5)$ & $(6)$ \\
\hline 129.550781 & 17.674994 & 97.0 & 74.2 & 27.8 & \\
129.540726 & 17.675444 & 1.3 & 3.2 & 0.0 & \\
129.549530 & 17.675861 & 2.5 & 4.6 & 0.0 & \\
129.551239 & 17.675165 & 3.1 & 5.5 & 0.0 & \\
129.562271 & 17.673082 & 5.7 & 14.5 & 0.0 & \\
\hline 129.545731 & 31.886414 & 82.6 & 25.2 & 27.6 & \\
129.542221 & 31.886194 & 3.8 & 10.2 & 0.0 & \\
129.546097 & 31.886360 & 7.6 & 7.8 & 1.0 & \\
129.546127 & 31.886318 & 17.7 & 0.1699 & 0.2 & -0.3 \\
129.550400 & 31.887083 & 1.9 & 9.6 & 0.0 & \\
\hline 129.555511 & 13.968682 & 38.1 & 137.2 & 52.8 & \\
129.543793 & 13.953083 & 21.9 & 26.5 & 0.0 & \\
129.554718 & 13.969610 & 8.6 & 8.8 & 1.0 & \\
129.554718 & 13.969643 & -18.7 & 2.0134 & 0.1 & 6.7 \\
129.568069 & 13.983055 & 14.5 & 17.5 & 0.0 & \\
\hline 129.567520 & 26.378452 & 1.2 & 44.8 & 115.5 & \\
\hline
\end{tabular}


Table. (Contd.)

\begin{tabular}{c|c|c|c|c|c}
\hline$\alpha_{2000}$ & $\delta_{2000}$ & \multicolumn{4}{|c}{ Characteristics of the source } \\
\hline$(1)$ & $(2)$ & $(3)$ & $(4)$ & $(5)$ & $(6)$ \\
\hline 129.567352 & 26.374390 & 13.7 & 10.9 & 0.0 & \\
129.567673 & 26.376307 & 19.2 & 53.7 & 0.0 & \\
129.567749 & 26.386833 & 36.0 & 51.0 & 0.0 & \\
\hline 129.580429 & 40.770386 & 14.7 & 29.5 & 18.5 & \\
129.579269 & 40.767113 & 2.4 & 7.3 & 0.0 & \\
129.580505 & 40.770500 & 1.9 & 3.1 & 0.0 & \\
129.582016 & 40.775028 & 1.7 & 8.1 & 0.0 & \\
\hline 129.586914 & 17.208118 & 73.0 & 43.1 & 90.5 & \\
129.581558 & 17.207390 & 8.3 & 33.1 & 0.0 & \\
129.585205 & 17.206223 & 2.5 & 13.1 & 0.0 & \\
129.593689 & 17.210306 & 10.9 & 44.3 & 0.0 & \\
\hline 129.595459 & 12.498256 & 80.2 & 18.8 & 179.5 & \\
129.592773 & 12.497806 & 29.0 & 35.6 & 0.0 & \\
129.595444 & 12.498250 & 44.7 & 51.2 & 1.0 & \\
129.595474 & 12.498255 & -19.1 & 1.6294 & 0.1 & -0.0 \\
129.598053 & 12.498694 & 78.5 & 92.7 & 0.0 & \\
\hline 129.610794 & 0.017126 & 21.9 & 59.2 & 29.7 & \\
129.607422 & 0.010861 & 6.0 & 8.9 & 0.0 & \\
129.610001 & 0.012806 & 6.2 & 7.9 & 0.0 & \\
129.614105 & 0.025889 & 11.2 & 12.9 & 0.0 & \\
\hline
\end{tabular}

The data for each radio source is arranged in blocks. The first line of each block describes the parameters of the object as a whole:

(1) average right ascension in degrees;

(2) average declination in degrees;

(3) position angle of the axis in degrees;

(4) angular size in arc seconds (we adopt the angular size to be the separation between the mutually most distant components of the radio source);

(5) integrated flux in mJy (the flux of a radio source is the sum of the integrated fluxes of its components). The three latter parameters are arranged into the column named "Characteristics of the source".

Every subsequent line gives the data on every component that makes up the radio source considered. These data are adopted from the FIRST catalog:

(1) right ascension in degrees;

(2) declination in degrees;

(3) flux of the unresolved component in mJy;

(4) integrated flux of the component in mJy;

(5) zero, if the component is not identified with any optical object, and one if such identification exists. Unity indicates that the next line contains the data on the optical object:
(1) right ascension in degrees;

(2) declination in degrees;

(3) V- or g-band magnitude. Negative magnitude means that the object is a quasar. If the absolute value of this parameter is equal to unity it means that we could not find either $\mathrm{V}$ - or g-band magnitude for the object;

(4) redshift;

(5) separation between the optical object and the corresponding radio component identified by the program, in arcsec;

(6) distance between the optical object and the axis of the radio source in arcsec.

The catalog in the above format is available at the web-page of the Special Astrophysical Observatory of the Russian Academy of Sciences at: ftp://ftp.sao.ru/cifs/cats/cats/FIRST_Amir/ /f1912kak5.txt.

\section{STATISTICS OF THE CATALOG}

Figure 2 shows the sky distribution of the radio sources of the catalog, which coincides with the sky area of the FIRST survey. Figure 3 shows the differential distribution of the fluxes of the radio sources of the catalog normalized to the "Euclidean statistics". Figure 4 shows the distribution of angular sizes of the radio sources. The resulting probability density of the distribution of angular sizes of the 


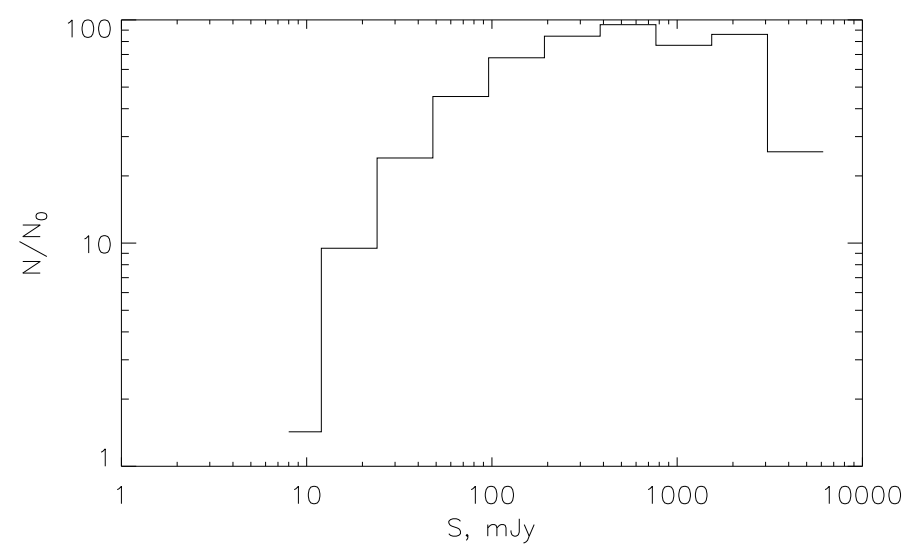

Figure 3. Differential flux distribution of the radio sources of the catalog normalized to the statistics in isotropic Euclidean space.

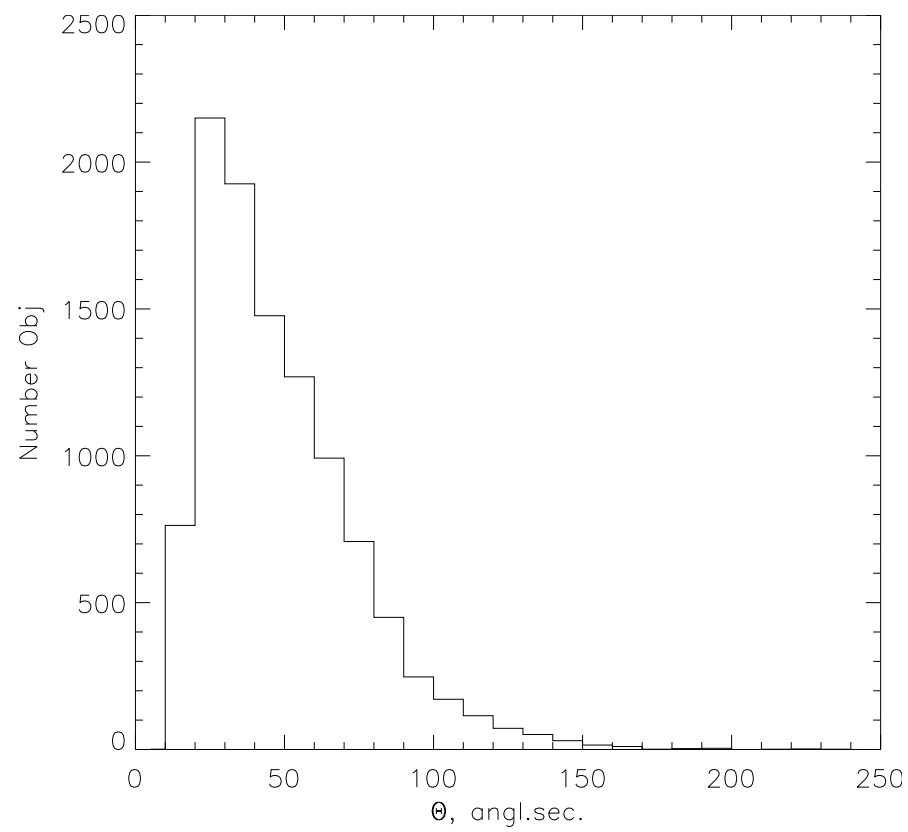

Figure 4. Distribution of angular sizes of radio sources.

radio sources of the catalog in the $20^{\prime \prime}-200^{\prime \prime}$ interval can be approximated fairly well by the following function:

$$
P(\theta) d \theta=0.025 e^{-0.000247(\theta-3.3)^{2}} d \theta .
$$

Figure 5 shows the distribution of the redshifts of radio sources identified: galaxies (the short-dashed line) and quasars (the solid line). This figure also shows the redshift dependence of the number of objects in terms of the Einstein-de Sitter model (the long-dashed line). It is evident that the cessation of the increase and subsequent rapid decline of the number of objects with increasing $\mathrm{Z}$ is due to the instrumental limitations of optical observations, and not to the actual decrease of the density of these objects. At small $\mathrm{Z}$, the computed dependence agrees fairly well with the experiment, and this should be viewed as an evidence of absence of any significant selection effects during the composition of the catalog. Of great interest is the "angular size-redshift" relation (Fig. 6). Here we also show the relations derived in terms of the Euclidean (the dashed line) and Einstein-de Sitter (the solid line) models for radio sources with the linear size of $500 \mathrm{kpc}$. Legg [14] and Miley [15] constructed this relation using objects of the 3CR catalog and showed that the upper envelope of the plot in angular size can be described fairly well in terms of the Euclidean model $(\theta \sim 1 / Z)$. To explain the discrepancy between this result and the main models of space, the above authors suggested that linear sizes of radio sources may evolve as $D \sim(1+Z)^{-1.5}$. Amirkhanyan [16] showed that the observed upper envelope of this plot can be ex- 


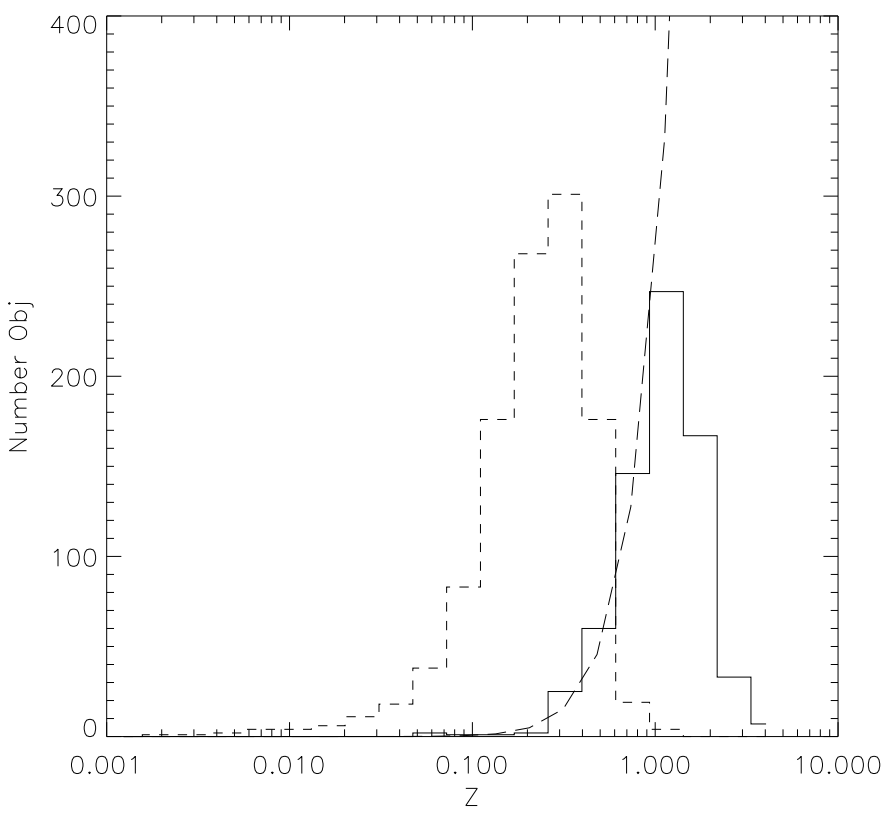

Figure 5. Redshift distribution of identified radio sources of the catalog. The short-dashed and solid lines show the distributions for galaxies and quasars, respectively. The long-dashed line shows the computed dependence for the Einstein-de Sitter model.

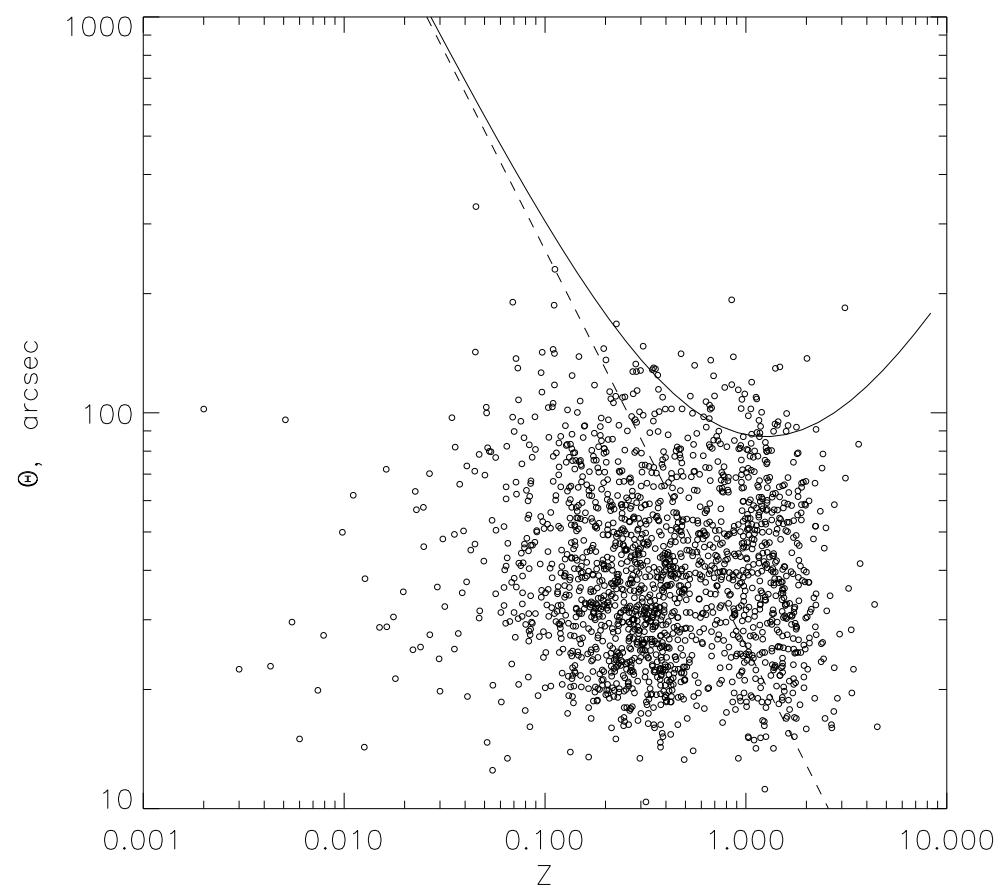

Figure 6. The "angular size-redshift" relation. Also shown here are the dependencies of radio sources with the linear size of $500 \mathrm{kpc}$ in the Euclidean (the dashed line) and Einstein-de Sitter (the solid line) models.

plained by the selection effect due to the limited sensitivity of the surveys and anisotropy of the directivity pattern of the radio sources. The detection threshold of the FIRST survey is three orders of magnitude lower than that of the 3CR survey, and therefore the selection boundary should have moved far upward in terms of redshift. It is evident from Fig. 6 that the upper limit of the new diagram agrees better with the standard model than with the Euclidean model. However, the parameters of the model are so far difficult to estimate even from the data for 1801 objects. 
(a)

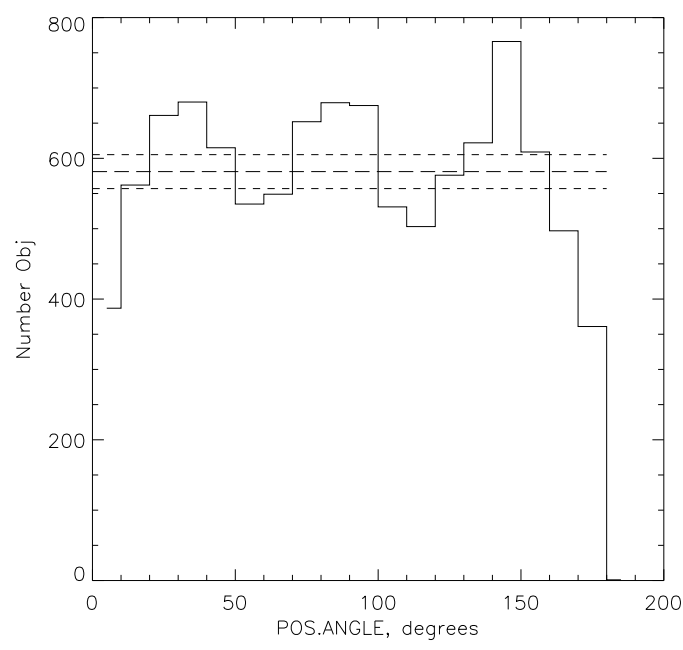

(c)

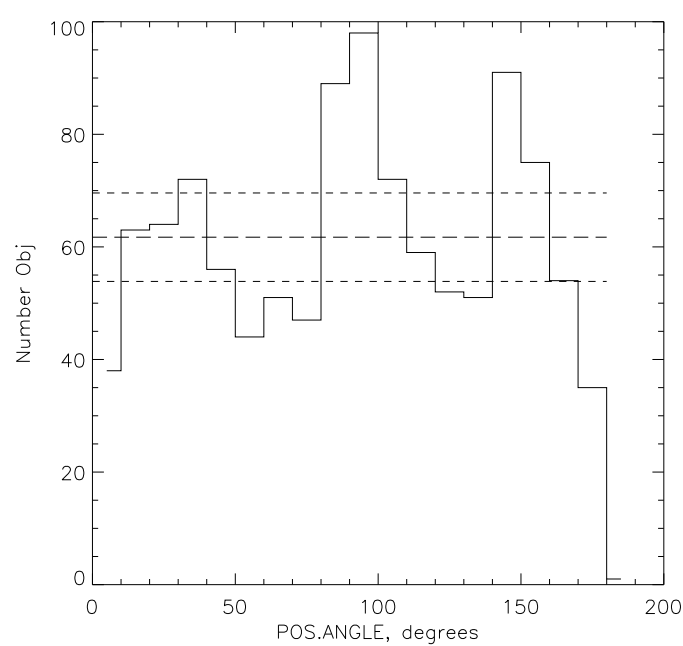

(b)

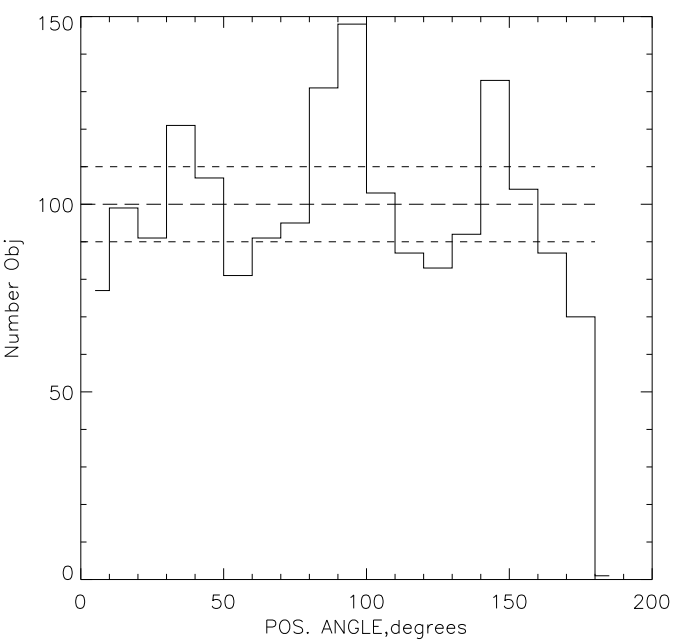

(d)

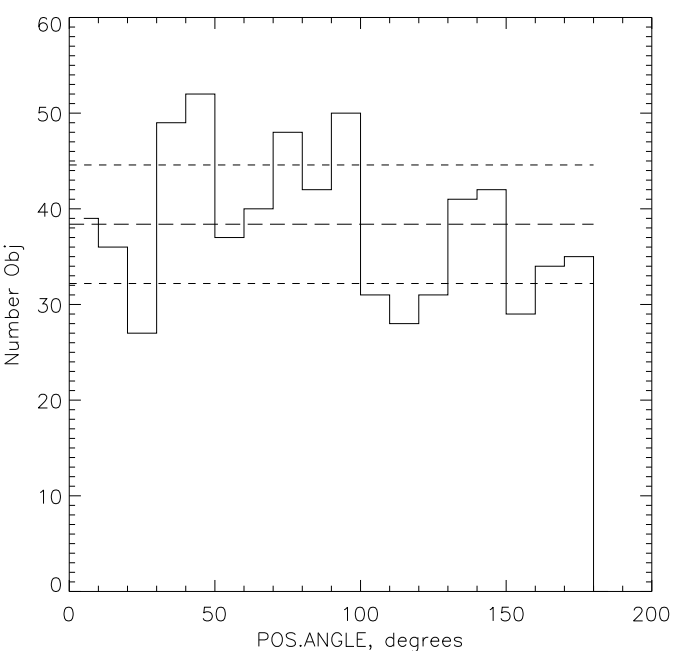

Figure 7. Distributions of position angles of the radio sources of the catalog. The dashed lines show the average level and the error interval under the assumption of equiprobable distribution of angles. Shown are: the distribution of the position angles of the radio sources of the catalog (a); the distribution of the position angles of the radio sources of the catalog with known Z (b); the distribution of the position angles of the radio sources of the catalog with known Z and identified with galaxies (c), and the histogram of the position angles of the radio sources of the catalog with known $\mathrm{Z}$ and identified with quasars (d).

\section{CONCLUSIONS}

Figure 7a shows the distribution of position angles of the axes of the radio sources. Its $\chi^{2}$ value is $\chi^{2}=314.4$, and the probability of it being isotropic is less than $10^{-7}$. This result inevitably implies that the spatial orientation of the axes of the radio sources is anisotropic. This naturally brings up the question where this anisotropy is located. The distribution of position angles of the axes of the radio sources with known redshifts (Fig.7b) is similar to the corresponding distribution for the entire sample. However, the distributions of the position angles of the radio sources with known redshifts identified with 1112 galaxies (Fig.7c) and 691 quasars (Fig.7d) differ appreciably from each other - galaxies exhibit a much stronger nonuniformity than quasars. Does this mean that galaxies are the main contributors to the nonuniformity of the distribution and anisotropy extends only out to $\mathrm{Z} \sim 0.5-1$ ? We do not yet know the answer to this question. And our second (cautious) conclusion is: the fact that the minimum of the histogram lies near $0^{\circ}$ means that the axes of the radio sources are mostly oriented in the direction of the equator. 


\section{ACKNOWLEDGMENTS}

The author is grateful to I. D. Karachentsev for fruitful discussion of this work.

1. F. G. Brawn, Monthly Notices Roy. Astronom. Soc. 99, 534 (1938).

2. F. G. Brawn, Monthly Notices Roy. Astronom. Soc. 127, 517 (1963).

3. P. Nilson, Ann. Uppsala astron. Observ. 6, 1 (1973).

4. A. Lauberts, The ESO/Uppsala Survey of the $\mathrm{ESO}(\mathrm{B})$ Atlas. (1982).

5. I. D. Karachentsev, V. E. Karachentseva, and S. L. Parnovsky, Astron. Nachr. 314, 97 (1993).

6. M. Reinhardt, Monthly Notices Roy. Astronom. Soc. 156, 151 (1972).

7. P. Nilson, Rep. Uppsala astron. Observ. 3, 1 (1974).

8. A. V. Mandzhos, A. Ya. Gregul', I. Yu. Izotova, and V. V. Tel'nyuk-Adamchuk, Astrofizika 26, 321 (1987).

9. S. L. Parnovsky, I. D. Karachentsev, and
V. E. Karachentseva, Monthly Notices Roy. Astronom. Soc. 268, 665 (1994).

10. V. R. Amirkhanian, Bull. Spec. Astrophys. Obs. 37, 119 (2000).

11. R. L. White, R. H. Becker, D. J. Helfand, and M. D. Gregg, Astronom. J. 475, 479 (1997).

12. http://cas.sdss.org/astro/en/tools/crossid/ /crossid.asp

13. M. P. Veron-Cetty and P. Veron, Astronom. and Astrophys. 455, 773 (2006).

14. T. H. Legg, Nature 226, 65 (1970).

15. G. K. Miley, Monthly Notices Roy. Astronom. Soc. 152, 477 (1971).

16. V. R. Amirkhanyan, Soobshchenija Spetsial'noi Astrofiz. Obs., 61, 112 (1989). 